\title{
Seminare / Séminaires / Seminari 2012
}

\section{Praxiseröffnung/-übernahme}

\section{Themen}

Juristische Aspekte (Praxisbewilligung, Zulassung zur Sozialversicherung, Vertragswesen), Gesellschaftsformen/Ehe- und Erbrecht (Trennung Privat- vom Geschäftsvermögen, Ehegüterstand, Erbschaftsplanung), Praxiseinrichtung (Inneneinrichtung, Kostenberechnung), Praxisadministration (Leistungserfassungs- und Abrechnungssysteme), Unternehmensbewertung einer Arztpraxis (Berechnung und Beurteilung des Unternehmenswertes), Finanzierung der Arztpraxis (Businessplan, Kredite, Absicherungsmöglichkeiten), Versicherungen/Vorsorge/Vermögen (Personen- und Sachversicherungen, Vorsorgeplanung).

\section{Sponsoren}

Die Kosten werden durch Sponsoren (www.fmhservices.ch) gedeckt.

\section{Daten}

$\begin{array}{lll}\text { K01 Donnerstag, 8. März 2012 } & \begin{array}{l}\text { Zürich } \\ \text { 9.00-16.30 Uhr }\end{array} & \text { FMT } \\ \text { K02 } & \text { Donnerstag, 3. Mai 2012 } & \begin{array}{l}\text { St. Gallen } \\ \text { 16.00-20.30 Uhr }\end{array} \\ \text { Hotel Radisson }\end{array}$

\section{Praxisübergabe}

Hinweis: Vor allem aus steuer- und vorsorgeplanerischer Sicht lohnt es sich, sich bereits frühzeitig (5-10 Jahre) mit diesem Thema auseinanderzusetzen.

\section{Themen}

Juristische Aspekte (Praxisübergabevertrag, allg. Vertragswesen, Übergabe der Krankengeschichten), Unternehmensbewertung einer Arztpraxis (Berechnung Inventarwert und Goodwill als Verhandlungsbasis), Versicherungen/Vorsorge/Vermögen (Übergabe/Auflösung von Versicherungsverträgen, Pensions- und Finanzplanung), Steuern (Steueraspekte bei der Praxisübergabe, Optimierung der steuerlichen Auswirkungen, Liquidations- und Grundstückgewinnsteuer, Bestimmung des optimalen Übergabezeitpunktes).

\section{Sponsoren}

Die Kosten werden durch Sponsoren (www.fmhservices.ch) gedeckt.

\author{
Daten \\ K06 Donnerstag, 15. März 2012 Zürich \\ 13.30-18.00 Uhr \\ FMT \\ K07 Donnerstag, 10. Mai 2012 \\ 16.00-20.30 Uhr \\ St. Gallen \\ Hotel Radisson
}

Finanz- und Steuerplanung

\section{Themen}

Finanzplanung (Businessplan, Buchhalterische Massnahmen vor Praxiseröffnung/ -übernahme, Standardkontenplan für Ärzte, System der doppelten Buchhaltung, EDVunterstützte Buchführungslösung), Steuern (Steueraspekte bei Eintritt in die Selbständigkeit, Steuerfallen und Steuerrisiken, optimierte Steuerplanung).

\section{Sponsoren}

Die Kosten werden durch Sponsoren (www.fmhservices.ch) gedeckt.

\section{Daten}

$\begin{array}{lll}\text { K11 Donnerstag, 22. März } 2012 & \begin{array}{l}\text { Zürich } \\ \text { 13.30-18.00 Uhr }\end{array} & \text { FMT } \\ \text { K12 } & \begin{array}{l}\text { Donnerstag, 20. September 2012 } \\ \text { 13.30-18.00 Uhr }\end{array} & \begin{array}{l}\text { Bern } \\ \text { Schmied- } \\ \text { stube }\end{array}\end{array}$

\section{Praxiscomputerworkshop}

Inhalt

Der Workshop beinhaltet eine Einführung in die Anforderungen an ein Praxisinformationssystem sowie Unterstützung im Evaluationsprozess. Anhand einer modernen vernetzten Praxisinfrastruktur werden die Beurteilungskriterien für eine praxisund zukunftstaugliche Softwarelösung dargestellt. Die Workshopteilnehmer/innen erhalten die einmalige Möglichkeit, sechs führende Praxisinformationssysteme (Leistungserfassung, elektronisches Abrechnen unter Einbezug der TrustCenter, Agendaführung, Statistiken, Laborgeräteeinbindung, elektronische Krankengeschichte, Finanzbuchhaltungslösungen usw.) kennenzulernen.

\section{Ziel}

Die Teilnehmer/innen erhalten einen Anforderungskatalog, welcher ihnen erlaubt, ihre Vorstellungen für ein modernes Praxisinformationssystem besser $\mathrm{zu}$ formulieren und diese dem Softwarehersteller zu dessen Vorbereitung zu kommunizieren. Zudem erhalten sie einen ersten Überblick über führende Softwarelösungen.

\section{Daten \\ K13 Donnerstag, 29. März 2012 Zürich 13.30-18.00 Uhr Technopark \\ K14 Donnerstag, 28. Juni 2012 Bern 13.30-18.00 Uhr BERNEXPO}

\section{Ouverture et reprise d'un cabinet médical}

\section{Contenu}

Business plan (préparation du plan de financement et crédit d'exploitation, financement par la banque), Aménagement (implantation, projet et concept d'aménagement, choix du mobilier, budget), Estimation d'un cabinet (inventaire et goodwill), Administration d'un cabinet médical (dans le cabinet, par la banque), Assurances (toutes les assurances à l'intérieur et autour du cabinet), Passage du statut de salarié à celui d'indépendant et fiscalité.

\section{Sponsors}

Les coûts sont pris en charge par divers sponsors (voir www.fmhservices.ch).

\section{Dates}

$\begin{array}{lll}\text { K20 Jeudi, } 8 \text { mars } 2012 & \text { Lausanne } \\ \text { 13.30-18.00 h } & \text { World Trade Center } \\ \text { K21 Jeudi, } 31 \text { mai } 2012 & \text { Genève }\end{array}$

K21 Jeudi, 31 mai 2012 Genève 13.30-18.00 h Crowne Plaza

\section{Remise d'un cabinet médical}

\section{Contenu}

Aspects juridiques (autour du contrat de remise/reprise), Estimation d'inventaire et goodwill d'un cabinet, Assurances (prévoyance, assurances à l'intérieur et autour du cabinet), Conséquences fiscales d'une remise.

\section{Sponsors}

Les coûts sont pris en charge par divers sponsors (voir www.fmhservices.ch). 


\section{Dates}

$\begin{array}{cl}\text { K24 Jeudi, } 10 \text { mai } 2012 & \text { Lausanne } \\ 17.00-21.30 \mathrm{~h} & \text { World Trade } \\ & \text { Center }\end{array}$

K25 Jeudi, 15 novembre 2012 Genève 17.00-21.30 h Crowne Plaza

Apertura e rilevamento di uno studio medico

\section{Contenuto}

Business plan (preparazione del piano di finanziamento e del credito d'esercizio, prestito bancario), Pianificazione (insediamento, progetto e pianificazione, scelta del mobilio, budget), Valutazione di uno studio medico (inventario e goodwill), Amministrazione di uno studio medico (interna allo studio, rapporti con la banca), Assicurazioni (tutte le assicurazioni necessarie interne ed esterne allo studio), Passaggio dallo stato di dipendente a quello di indipendente, fiscalità.

\section{Documentazione}

Basato su una check-list per l'apertura di uno studio medico, il raccoglitore, consegnato ad ogni partecipante, contiene tutte le informazioni utili durante e dopo il seminario.

\section{Seminarsponsoren 2012}

Die Unterstützung durch verschiedene Sponsoren ermöglicht es der FMH Consulting Services AG, ihre Seminarreihen für FMH Services Mitglieder teils kostenlos, teils kostengünstig anzubieten. Gerne stellen wir Ihnen diese Firmen in einem Kurzporträt vor.

\section{medics labor}

\section{professionell und persönlich}

Medics Labor AG

Chutzenstrasse 24, 3001 Bern

Tel. 03137220 02, Fax 0313714044

info[at]medics-labor.ch

www.medics-labor.ch

\section{Medizinisches Labor und mehr}

Medics Labor ist ein Schweizer Unternehmen, zu Hause in Bern, hier verwurzelt und seit vielen Jahren erfolgreich tätig im Kanton sowie weiteren Regionen.

Geschätzt als persönliches, unkompliziertes Gegenüber, überzeugt Medics Labor durch fachliches und menschliches Gespür mit zahlreichen Hilfestellungen und Dienstleistungen. Wir verstehen uns als sozialer Arbeitgeber und beschäftigen auch behinderte Personen.

\section{Sponsor}

Diversi sponsor si fanno carico delle spese (si rimanda al sito www.fmhservices.ch).

\section{Date}

\begin{tabular}{|c|c|c|}
\hline K50 & $\begin{array}{l}\text { Martedì, } 6 \text { marzo } 2012 \\
\text { dalle } 17.00 \text { alle } 21.00\end{array}$ & $\begin{array}{l}\text { Chiasso } \\
\text { FMH } \\
\text { Fiduciaria } \\
\text { Services }\end{array}$ \\
\hline K51 & $\begin{array}{l}\text { Mercoledi, } 16 \text { maggio } 2012 \\
\text { dalle } 14.00 \text { alle } 18.00\end{array}$ & $\begin{array}{l}\text { Chiasso } \\
\text { FMH } \\
\text { Fiduciaria } \\
\text { Services }\end{array}$ \\
\hline
\end{tabular}

\section{Anmeldung und Auskunft /}

Inscription et information /

Iscrizioni e informazioni

www.fmhservices.ch oder FMH Consulting Services, Cornelia Steinmann, Burghöhe 1, 6208 Oberkirch, Tel. 04192500 77, Fax 041 9210586.

\section{Hinweis / Remarque / Osservazioni}

Bei sämtlichen Seminaren, bei denen die Kosten teilweise oder gänzlich von Seminarsponsoren gedeckt werden, werden die Teilnehmeradressen den jeweiligen Sponsoren zur Verfügung gestellt.

Medics Labor ist ein Labor von Ärzten für Ärzte. Es gehört den Laborspezialisten und den Ärzten, die das Unternehmen gemeinsam führen.

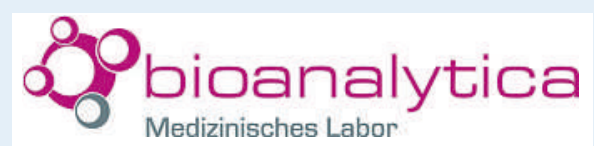

Bioanalytica AG

Maihofstrasse 95a, 6006 Luzern

Tel. 04142931 31, Fax 0414293130

service[at]bioanalytica.ch

www.bioanalytica.ch

\section{Engagierte Kompetenz}

Bioanalytica, 1957 in Luzern gegründet, basiert auf einer langjährigen Tradition. Stetige Innovation und ein Team qualifizierter Fachspezialisten und Labormediziner bilden das Fundament unserer Kompetenz. Qualität und Seriosität - das sind die Werte, denen wir uns verschrieben haben. Aus der Überzeugung, dass dies auch unseren Kunden wesentliche Vorteile bietet, haben wir unser Labor im Jahre 2000 akkreditieren lassen.

Schnell, wenn notwendig auch rund um die Uhr, sind wir für Sie da. Mit dem Know-
Les adresses des participants aux séminaires dont les coûts sont couverts en partie ou totalement par des sponsors sont communiquées aux sponsors concernés.

Gli indirizzi dei partecipanti ai seminari, i cui costi sono coperti in parte o completamente da degli sponsor, vengono comunicati agli sponsor interessati.

\section{Annullierungsbedingungen /}

\section{Conditions d'annulation /}

\section{Condizioni d'annullamento}

Bei Abmeldungen oder Fernbleiben werden folgende Unkostenbeiträge erhoben:

Un montant est perçu pour une absence ou une annulation. Il est de:

Un importo verrà rimborsato in caso di assenza o annullamento. Esso sarà di:

- 50 CHF pro Person ab 14 Tage vor Seminarbeginn / par personne dans les 15 jours avant le début du séminaire/ per persona entro i 15 giorni prima dell'inizio del seminario

- 100 CHF pro Person ab 7 Tage vor Seminarbeginn oder Fernbleiben / par personne dans les 7 jours avant le début du séminaire / per persona entro i 7 giorni prima dell'inizio del seminario.

how von rund 85 Mitarbeitenden, modernster Laborautomation und Informationstechnologie sind unsere Laborresultate in kürzester Zeit verfügbar.

Bei Bioanalytica stehen Sie als Kunde im Mittelpunkt. Wir unterstützen Sie und Ihr Praxisteam jederzeit gerne optimal im persönlichen Kontakt und mit zahlreichen wertvollen zusätzlichen Dienstleistungen.

\section{$\overbrace{1}$ ANALYTICA \\ MEDIZINISCHE LABORATORIEN AG}

Analytica Medizinische Laboratorien AG Falkenstrasse 14

8024 Zürich

Tel. 0442505050

kundendienst[at]analytica.ch

\section{Werte. Verbinden.}

Sehr geehrte Frau Kollega, sehr geehrter Herr Kollege

Die Analytica Medizinische Laboratorien AG wurde 1957 von meinem Vater gegründet, und ich durfte das Labor 1985 in zweiter Generation übernehmen. Eigentlich mag ich 
es gar nicht, mich unpersönlich und mit schönen Worten vorzustellen. Ich bin durch und durch Praktiker, gibt es ein Problem, dann löse ich es, und kennt mich ein Kunde noch nicht persönlich, dann komme ich gerne vorbei und stelle mich vor.

Wir haben uns nach langen Diskussionen für den Slogan «Werte. Verbinden.» entschieden. Der Punkt zwischen den Worten ist kein Schreibfehler. Wir haben Werte. Werte, die uns einen persönlichen Umgang miteinander ermöglichen, Werte, die die Qualität unserer Arbeit beschreiben, und Werte, die wir mit Ihnen - unseren Kunden - teilen. Diese Werte verbinden uns miteinander und stellen das tragfähige Netz dar, das uns alle seit so vielen Jahren trägt. Für das bin ich dankbar.

Dr. med. Peter Isler

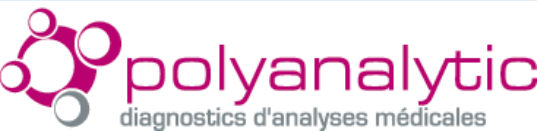

Polyanalytic SA

Avenue de Sévelin 18, 1004 Lausanne

Tel. 02180492 50, Fax 0218049250

info[at]polyanalytic.ch

www.polyanalytic.ch

Polyanalytic ist ein Labor für medizinische Analysen, das auf dem Gebiet der Kantone Waadt und Neuenburg tätig ist.

Gestützt auf seine Kompetenzen, die es in den Dienst der Patientinnen und Patienten und der Ärzteschaft stellt, bietet Polyanalytic eine umfassende Palette von medizinischen Analysen.

Seit Polyanalytic 1983 gegründet wurde, ist das Unternehmen für herausragende Qualität und kundennahe Dienstleistungen bekannt. Den frei praktizierenden Ärztinnen und Ärzten werden mit unvergleichlicher Konstanz verlässliche, rasche und kompetente Leistungen geboten, damit sie ihre Kunst ausüben können.

Mit Polyanalytic verfügen die Ärztinnen und Ärzte nicht nur über einen Partner, der auf ihre Bedürfnisse eingeht, sondern auch tagtäglich über echte Unterstützung bei ihrer Tätigkeit.

Polyanalytic ist mehr als ein Unternehmen: Dank der Kompetenz der Menschen, die dort arbeiten, kann die Ärzteschaft darauf vertrauen, dass bei den Patientinnen und Patienten, für die sie verantwortlich ist, optimale Laborkontrollen gewährleistet sind.

\section{0: dianalabs}

Dianalabs SA

Rue de la Colline 6, 1205 Genève

Tel 02280712 40, Fax 0228071244

info[at]dianalabs.ch

www.dianalabs.ch

Dianalabs ist ein Labor für medizinische Analysen, das 1988 mit dem Ziel gegründet wurde, der Ärzteschaft und den Patientinnen und Patienten optimale Laborkontrollen $\mathrm{zu}$ bieten.

Aufgrund seiner Publikationen und Präsentationen ist das Genfer Labor für die Qualität seiner Serologie international anerkannt.

Wir bieten eine umfassende Palette von medizinischen Analysen, um alle Bedürfnisse der Medizin abzudecken. Doch Dianalabs ist mehr als ein polyvalentes Allround-Labor: Dank seinem Spezialistenteam deckt es eine Vielzahl von Fachgebieten ab und bietet eine Schnittstelle zu jedem medizinischen Fachgebiet mit seinen besonderen Bedürfnissen.

Durch die wissenschaftliche Zusammenarbeit mit den Ärztinnen und Ärzten und den universitären Zentren wurde uns klar, dass nur ein regionales Unternehmen, das grundlegende menschliche Werte wie Qualität, Austausch und Dienstleistungsbereitschaft in den Vordergrund stellt und mit den lokalen Verhältnissen vertraut ist, die Bedürfnisse der Bevölkerung und der Ärzteschaft kompetent erfüllen kann.

\section{Schmid MEDIPRINT • CLASSICPRINT}

Schmid Mogelsberg AG, Ärztedrucksachen Sonnmattstrasse 1, 9122 Mogelsberg Tel. 07137560 80, Fax 0713756081 info[at]schmid-mogelsberg.ch www.schmid-mogelsberg.ch

Bei der Gestaltung von zweckmässigen Arztformularen (Patientenkarten, KG-Einlageblätter, usw) profitieren Sie von unserer langjährigen Erfahrung. Die Vergangenheit hat gezeigt, dass sich kaum zwei Ärzte für den gleichen Druck entscheiden. Auf Wunsch versenden wir eine individuell auf Ihre Fachrichtung zusammengestellte Druckmusterkollektion.

Testen Sie unsere praktischen Vordruckformulare!
An über 9000 Ärzte liefern wir Drucksachen, Papiere und Büroartikel für den Praxisalltag.

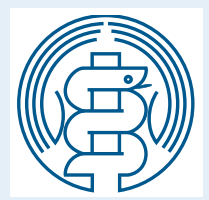

Schweizerische Ärzte-Krankenkasse Oberer Graben 37, Postfach 2046 , 9001 St. Gallen Tel. 07122718 18, Fax 0712271828 info[at]saekk.ch

www.saekk.ch

Die richtige Adresse für Erwerbsausfalldeckungen, Kollektivkrankenkasse und Versicherungsplanung

Mit mehr als 100 Jahren Erfahrung kennt unsere Organisation auch heute die Bedürfnisse der Ärztinnen und Ärzte. Sie bietet entsprechend durchdachte und kostengünstige Lösungen an, sowohl für Praxiseröffner/innen wie auch für selbständige und angestellte Ärztinnen und Ärzte.

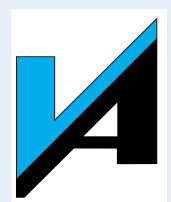

Versicherung der Schweizer Ärzte

Genossenschaft

Länggassstrasse 8, 3000 Bern 9

Tel. 03130125 55, Fax 0313025156

versa[at]versa.ch

www.versa.ch

Spezialisiert auf die Bedürfnisse von Ärztinnen und Ärzten und deren Ehegatten, bietet die Versicherung der Schweizer Ärzte Genossenschaft individuelle, den jeweiligen Bedürfnissen angepasste Versicherungslösungen im Bereich der privaten Vorsorge an.

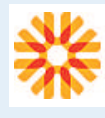

\section{Unilabs}

www.unilabs.ch

\section{UNILABS - Ihr Qualitätslabor}

schnell, zuverlässig und nah

Unilabs ist im Bereich der medizinischen Analysen ein kompetenter, transparenter und zuverlässiger Partner. Wir bieten Ihnen überall in der Schweiz ein komplettes Analysenspektrum, umfassende Dienstleistungen und 
kompetente Fachberatung an. In der Deutschschweiz sind dies Unilabs Mittelland mit den Standorten Basel, Bern, Langnau, Solothurn, Thun; Unilabs Zürich und Unilabs Dr. Weber. Unilabs bietet Weiterbildungen für das gesamte Praxis-Team sowie Beratung bei Praxis- und Laborbedarf.

Unsere vielseitigen und regionalen Dienstleistungen basieren auf einer fundierten wissenschaftlichen Struktur und hochstehenden Qualität.

Unilabs Schweiz - aktuell 900 Mitarbeiterinnen und Mitarbeiter, 72 Wissenschaftler und 22 Labors in Ihrer Nähe.

\section{medica}

\section{MEDIZINISCHE LABORATORIEN Dr. F. KAEPPELI AG}

\section{MEDIZINISCHE LABORATORIEN}

DR. F. KAEPPELI AG

Eidgenössisch anerkannte Laboratorien

Wolfbachstrasse 17, 8024 Zürich

Tel. 04426999 99, Fax 0442699909

info[at]medica-labor.ch

www.medica-labor.ch

Der promovierte Mikrobiologe und Biochemiker Dr. F. Käppeli, Laborspezialist FAMH, übernahm 1976 das heute über 50-jährige Unternehmen und gründete als dessen Leiter und Inhaber die Einzelfirma medica. Der wichtigste unternehmerische Leitgedanke von Dr. F. Käppeli heisst kontinuierliche Innovation und Schaffung wegweisender Standards auf allen Gebieten der Labormedizin: Mikrobiologie inklusive Parasitologie, Serologie, Immunologie, klinische Chemie, Hämatologie, molekulare Diagnostik und Pathologie in Human- und Veterinärmedizin. So entstand ein Kompetenz-Zentrum für Labordiagnostik von gesamtschweizerisch grosser Bedeutung. Die modernst ausgebauten Laboratorien werden laufend erweitert und befinden sich im Herzen von Zürich. Über 200 Angestellte der Partnerlabors, begleitet von Spezialisten aus Medizin, Pharmakologie, Naturwissenschaften und Technik, garantieren für höchste Professionalität.

\section{IVF HARTMANN AG}

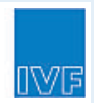

IVF HARTMANN AG

Victor-von-Bruns-Strasse 28,

8212 Neuhausen

Tel. 05267431 11, Fax 0526727441

info[at]ivf.hartmann.info

www.ivf.hartmann.info
Die IVF HARTMANN AG ist einer der führenden Anbieter für medizinische Verbrauchsgüter im Bereich Heilung, Pflege und Hygiene in der Schweiz. Ihre Lösungen helfen überall dort, wo Menschen geholfen wird. $\mathrm{Zu}$ ihren Kunden zählen somit Spitäler, Altersund Pflegeheime, Spitex-Organisationen, niedergelassene Ärzte, Apotheken, Drogerien und der Lebensmitteleinzelhandel. Das breite Angebot der IVF HARTMANN AG umfasst über 2000 Produkte - vom therapeutisch wirksamen Pflaster (z.B. Isola ${ }^{\circledR}$ Capsicum N) über funktionelle Verbände bis hin zu Produkten für die moderne Wundbehandlung (z.B. TenderWet $^{\circledR}$ oder CompriGel $^{\circledR}$ ) und Erste Hilfe (z.B. DermaPlast ${ }^{\circledR}$ ). Die IVF HARTMANN GRUPPE ist eine 60-prozentige Tochtergesellschaft der PAUL HARTMANN AG mit Sitz in Heidenheim an der Brenz (D) und beschäftigt rund 350 Mitarbeiter. Neben ihrem Hauptsitz in Neuhausen am Rheinfall (SH) verfügt die IVF HARTMANN AG über weitere Produktionsstätten in Gommiswald (SG) und Netstal (GL).

\section{mepha}

Mepha Pharma AG

Dornacherstrasse 114, 4147 Aesch

Tel. 0617054343

www.mepha.ch

\section{Mepha - wir setzen Massstäbe}

Mepha, die führende Generika-Herstellerin der Schweiz, steht im 7. Jahrzehnt ihrer denkwürdigen Erfolgsgeschichte. Unseren Beitrag zu wirksamer Prophylaxe und Therapie sehen wir in der Vermarktung von günstigen, gutverträglichen und hochwertigen Generika und Markengenerika, die nach modernsten Hightechverfahren und nach höchstem Schweizer Qualitätsstandard produziert werden. Unsere innovativen, kreativen Lösungen begeistern unsere Kunden immer wieder aufs Neue: zum Beispiel neuartige und verbesserte Anwendungsformen unserer Medikamente, die den Behandlungserfolg und das Wohlbefinden von Patientinnen und Patienten steigern. Alle unsere Leistungen gründen auf einer ganzheitlichen Sicht, welche die Interessen unserer Kunden, Mitarbeiter und Aktionäre, aber auch jene der übrigen Anspruchsgruppen in den Mittelpunkt stellt. Erstklassige Produkte, ein komplettes Package gefragter Dienstleistungen und offene Kommunikation sind weltweit Basis der Zufriedenheit unserer Kunden.

\section{EGalexis}

Galexis AG

Industriestrasse 2, Postfach, 4704 Niederbipp

Tel. 05885171 11, Fax 0588517114

info[at]galexis.com

www.galexis.com

Als Vollgrossist setzt Galexis AG Standards im Schweizer Gesundheitsmarkt. Wir beliefern unsere Kunden ganz nach dem Motto «Alles aus einer Hand» mit Pharma, Praxis- und Laborbedarf sowie Medizintechnik und erbringen darüber hinaus integrierte Dienstleistungen in der Gesundheitslogistik - schweizweit. Mit erprobten Lösungen fördert Galexis den Erfolg ihrer Kunden.

Möchten Sie ausserdem Ihre eigene Praxis praktisch, funktionell und ästhetisch einrichten? Genau hier kann Sie Galexis mit ihren Fachpartnern und einer langjährigen Erfahrung professionell beraten und unterstützen!

Überzeugen Sie sich - mit Galexis können Sie rechnen!

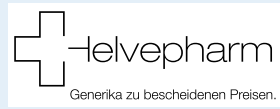

Helvepharm AG

Walzmühlestrasse 48, 8500 Frauenfeld Tel. 05272328 50, Fax 0527232858 info[at]helvepharm.ch www.helvepwharm.ch

Als Vermarkter und Vertreiber hochwertiger Generika tun wir alles dafür, um mit Innovationsgeist und Qualität eine führende Rolle im Schweizer Markt zu erreichen.

Helvepharm setzt auf preiswerte Generika. Auf Medikamente, die sich in Wirkstoff, Darreichungsform und Dosierung an die Originalpräparate anlehnen und mit diesen austauschbar sind. Auch unsere Arzneimittel werden durch Swissmedic auf Herz und Nieren geprüft. Helvepharm Generika sind gleich wirksam wie das Original, jedoch ungleich günstiger.

Mit über 60 Wirkstoffen in über 330 Darreichungsformen bieten wir kluge Alternativen zu Antihypertonika, Antidepressiva, Lipidsenkern, Gastrotherapeutika und für weitere relevante Gebiete. Helvepharm ist die günstige Basis im Gesundheitswesen.

Helvepharm in Frauenfeld gehört zu Zentiva und damit zum drittgrössten Generikaanbieter in Europa. Zentiva ist Teil der sanofi-aventis-Gruppe. 


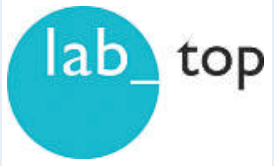

LabTop Medizinische Laboratorien AG Chriesbaumstrasse 6, 8604 Volketswil Tel. 04339930 30, Fax 0433993031 info[at]labtop.ch www.labtop.ch

\section{Das externe Labor in Ärztehand}

Ärzte halten zusammen: LabTop ist ein von Ärzten aufgebautes und von LaborProfis geführtes Privatlabor. Seit 10 Jahren unterstützt es den Arzt in seinem Sinne. LabTop ist überwiegend im Besitz von Ärzten und steht beteiligungswilligen Ärzten weiterhin offen.

Zur Selbsthilfe von praktizierenden Ärzten gestartet, klein, modern, unabhängig, exakt - typisch schweizerisch eben - bietet LabTop bestechend einfache und modernste Lösungen für die Arztpraxis. Bei LabTop bleibt die externe Analytik in Ärztehand. Vorteile für Sie: Als Novum bietet LabTop ein web-basiertes Geschäftsmodell, von dem Sie in verschiedener Hinsicht profitieren: Ressourcen-Einsparungen in Ihrer Praxis dank optimierter Prozesse, top Service, Messqualität, die höchsten Ansprüchen genügt, und nicht zuletzt, Ihr Beitrag bei der Analyseerfassung ist LabTop etwas wert. LabTop - von Ärzten aufgebaut, für Ärzte geführt.

\section{zur Rose}

Zur Rose Suisse AG

Walzmühlestrasse 60, 8500 Frauenfeld

Tel. Ärztegrossist 0527240020

Tel. Versandapotheke 0848842842

info[at]zur-rose.ch

www.zur-rose.ch

Zur Rose - zuverlässiger und vertrauensvoller Partner von mehr als 3000 Ärztinnen und Ärzten

Zur Rose ist Marktführer in der Schweizer Medikamentendistribution in den Bereichen Medikamentenversand und Arztpraxisbelieferung. Als standeseigenes Unternehmen vertritt Zur Rose die Interessen der Ärzteschaft.

\section{Zur Rose für Ärzte}

Mehr als 3000 Arztpraxen in der ganzen Schweiz erhalten von Zur Rose alles geliefert, was sie täglich benötigen: Medikamente,
Impfstoffe, Insuline, Seren, Magistralitäten, Verbrauchsmaterialien, Labor- und Röntgenbedarf sowie Praxisgeräte und Instrumente aller Art.

\section{Zur Rose für Patienten}

Im Auftrag der Ärztinnen und Ärzte verschickt die Versandapotheke Zur Rose portofrei Medikamente an über 200000 Kundinnen und Kunden. Zur Rose führt die Medikamenten- oder Bezugschecks kostenfrei aus und gewährt zusätzlich bis zu 12\% Rabatt.

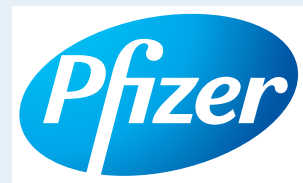

Pfizer AG

Schärenmoosstrasse 99, 8052 Zürich

Tel. 04349571 11, Fax: 0434957280

info.ch[at]pfizer.com

www.pfizer.ch

\section{Pfizer: Gemeinsam für eine gesündere}

Welt

Pfizer steht für die erfolgreiche Erforschung und Entwicklung von innovativen Arzneimitteln - und das schon seit über 160 Jahren. Damals wie heute gilt, dass die grossen medizinischen Herausforderungen keinen Aufschub dulden. Mit pharmazeutischen Innovationen leistet Pfizer einen wichtigen Beitrag zur Prävention und zur erfolgreichen Behandlung von weitverbreiteten, aber auch von seltenen Erkrankungen.

Als weltweit führendes forschendes Pharmaunternehmen setzen wir bei Pfizer unsere wissenschaftliche Kompetenz und unsere weltweiten Möglichkeiten ein, um die Lebensqualität und Gesundheit in jedem Lebensabschnitt zu verbessern.

In der Schweiz ist Pfizer seit 1959 ansässig. Mit rund 300 Mitarbeitenden und einem Umsatz von rund 400 Millionen Schweizer Franken (2011) gehört die Pfizer AG zu den bedeutendsten Pharmaunternehmen des Landes.

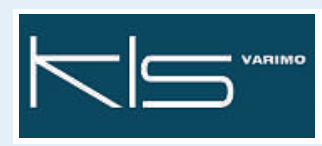

\section{KLS Müller AG}

Hertistrasse 24, 8304 Wallisellen

Tel. 04483950 40, Fax 0448395059

info[at]varimo.ch

www.varimo.ch

\section{KLS: drei Buchstaben - und dahinter} ein Familienbetrieb, der das Gute besser macht ...

Schon seit 1964, als Josef Müller eine bestehende Möbelschreinerei in Wallisellen übernahm, pflegen und leben wir das Schreinerhandwerk auf solidem Niveau. Im Laufe der Zeit machte sich die KLS Müller AG einen guten Namen im Küchenund Ladenbau sowie auch im Bereich der Klinik- und Praxiseinrichtungen.

Der Innovations- und Erfindergeist im Hause KLS Müller AG bringt immer wieder neue Ideen und Anwendungen zutage, so auch die jüngste, das modulare Ärztemöbel VARIMO. KLS: eine starke Marke für handwerklich präzise und ästhetisch ansprechende Räume: KLS Kennen Lohnt Sich.

Wer bei uns seine Wünsche, Vorstellungen, Pläne und Erwartungen plaziert, kann davon ausgehen, dass sie mit Lösungen beantwortet werden, die uns ebenso viel Freude bereiten wie unseren Kunden.

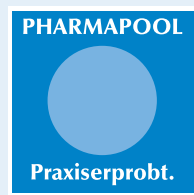

Pharmapool AG

Unterlettenstrasse 18, 9443 Widnau

Tel. 07172725 25, Fax 0717272555

info[at]pharmapool.ch

www.pharmapool.ch

Pharmapool ist der ärzteeigene Grossist mit 24-Stunden-Lieferbereitschaft. Das Kennen der Bedürfnisse von Arzt und Praxispersonal und das Wissen über die medizinischen Abläufe stehen im Mittelpunkt.

Dank der fundierten Betreuung und rationellen Belieferung wird die Wirtschaftlichkeit der von Pharmapool bedienten Praxen verbessert - sowohl von rezeptierenden als auch selbstdispensierenden Ärzten. Unabhängig von Einzelinteressen der PharmaBranche erhalten unsere Kunden das gesamte Sortiment an Originalprodukten, Generika, Seren, Verbrauchsmaterialien, Einrichtungsgegenständen und MiGeL-Artikeln zu transparenten Konditionen. Eng verbunden mit der attraktiven Lieferpolitik sind unsere praxiserprobten Dienstleistungen rund um die Medikamenten-Logistik, wie z.B. modernes Bestellwesen sowie praktische Preisnachführung und hilfreiche Einkaufs-Statistiken. Pharmapool - von Ärzten für Ärzte. 